\title{
AVALIAÇÃO DO USO DAS FERRAMENTAS DE GERENCIAMENTO DE PROJETOS
}

\section{EVALUATING THE USE OF PROJECT MANAGEMENT TOOLS}

\begin{abstract}
Antonio Cesar Amaru Maximiano Professor associado da Faculdade de Economia, Administração e Contabilidade da Universidade de São Paulo/ Brasil Supervisor de programas e projetos da Fundação Instituto de Administração/Brasil maximin@usp.br
\end{abstract}

Daniel Leroy Professeur des Universités, Directeur du Master 2, Recherche en Management,Management de la Qualité e des Projets, Institut d'Administration des Entreprises, Université François Rabelais,

Tours/França daniel.leroy@univ-tours.fr

Carlos Henrique Bittencourt Morais Mestrando do Programa de Pós-Graduação em Administração da Faculdade de Economia, Administração e Contabilidade da Universidade de São Paulo/ Brasil carlos.morais@usp.br

Elke Irene Buergers Mestranda do Programa de Pós-Graduação em Administração da Faculdade de Economia, Administração e Contabilidade da Universidade de São Paulo/ Brasil

buergers@usp.br

Márcio Roberto Moran Mestre em Administração pela Universidade de São Paulo/Brasil marcio.moran@usp.br

Ricardo Toshio Yugue Mestrando do Programa de Pós-Graduação em Administração da Faculdade de Economia, Administração e Contabilidade da Universidade de São Paulo/ Brasil yugue@usp.br

Editor Científico Responsável Prof ${ }^{a}$. Dr ${ }^{a}$. Simone Fernandes Queiroz Departamento de Administração da Pontifícia Universidade Católica de Minas Gerais/Brasil

Submissão: $27 / 02 / 2011$ Aprovação: 08/11/2011

\section{Resumo}

Neste trabalho, os autores relatam um estudo que teve o objetivo de identificar a frequência de utilização de ferramentas de gerenciamento de projetos. Os autores começaram selecionando na literatura um total de 47 ferramentas. Em seguida, um questionário usando uma escala do tipo Likert foi construído e submetido a uma amostra de gerentes de projetos, produzindo 168 questionários preenchidos, dos quais 64 por profissionais da construção civil e 104 por profissionais de outras áreas. Os resultados mostram que muitas ferramentas não são utilizadas, ou são muito pouco utilizadas, mas 
isso depende do conhecimento e da proficiência do usuário. O estudo, no entanto, não procurou medir o grau de conhecimento sobre as ferramentas, apenas sua utilização, com base nas respostas, uma vez que os respondentes eram profissionais da administração de projetos. Os resultados também mostram sutis diferenças entre as práticas dos projetos de construção e os das demais áreas de aplicação. Essas diferenças ensejam uma discussão sobre a ideia da complexidade como variável determinante da escolha das ferramentas e da abordagem de gerenciamento dos projetos.

Palavras-chave: Projeto. Ferramentas de gerenciamento de projetos. Complexidade.

\begin{abstract}
The authors report in this paper a study aimed at identifying the frequency of use of project management tools. The author began by selecting 47 tools in the literature. A Likert-type questionnaire was subsequently prepared and submitted to a sample of project managers, yielding 168 responses. Sixty-four of these were provided by construction professionals and 104 by professionals from other areas, especially consulting, information technology and product development. The results show that many tools are not used or are underused, but this depends on the knowledge and expertise of project managers. The study, however, sought only to measure the frequency of use, without taking into account the knowledge of the project manager. The results also show subtle differences between construction projects and those of other application areas. These differences allow for a discussion on the impact of complexity on project management practices.
\end{abstract}

Keywords: project, project management tools, complexity. 


\section{INTRODUÇÃO}

A administração de projetos é um corpo de conhecimentos que adquiriu o status de disciplina na esteira dos grandes empreendimentos da exploração espacial e da defesa nos anos 50-60. Subsequentemente, a emergência e o rápido crescimento de novos ramos de negócios e áreas do conhecimento - tecnologia da informação, qualidade total, produção enxuta, P\&D, Fórmula I, Jogos Olímpicos - que exigem uma abordagem de trabalho sob encomenda, deram um impulso adicional a essa disciplina. Essa evolução resultou na popularização de diversas técnicas, conhecidas como ferramentas de gerenciamento de projetos, orientadas para resolver os problemas práticos de objetivos, prazos, custos, pessoas e muitas outras variáveis dos projetos. Elas se juntaram a ferramentas antigas, de comprovada utilidade, como o gráfico de Gantt. No entanto um gerente de projetos, interessado em buscar soluções nos manuais, não encontrará muitas referências sobre a frequência de uso, para não falar da aplicabilidade da maioria dessas ferramentas. Ou seja, uma questão importante é: gerenciando projetos, que ferramentas as pessoas envolvidas efetivamente utilizam?

No estudo relatado neste trabalho, os autores procuraram responder a essa pergunta. O objetivo do estudo, portanto, foi identificar a frequência de utilização de ferramentas de gerenciamento de projetos. A premissa subjacente é que a frequência de utilização indica a utilidade dessas ferramentas. Assim, as ferramentas mais utilizadas são, presumivelmente, as mais úteis.

Os autores começaram selecionando na literatura um total de 47 ferramentas. Um questionário usando uma escala do tipo Likert foi construído e submetido a uma amostra de gerentes de projetos, produzindo 168 questionários preenchidos. Desse total, 64 foram respondidos por profissionais da construção civil e 104 por profissionais de outras áreas, principalmente consultoria e tecnologia da informação. Os resultados mostram que muitas ferramentas não são utilizadas, ou são muito pouco utilizadas, mas os autores estão conscientes de que isso depende do conhecimento e da proficiência do usuário. $\mathrm{O}$ estudo, no entanto, não procurou medir o grau de conhecimento sobre as ferramentas, apenas sua utilização, com base nas respostas, uma vez que os respondentes eram profissionais da administração de projetos. Os resultados também mostram sutis diferenças entre as práticas dos projetos de construção e os das demais áreas de aplicação. Essas diferenças ensejam uma discussão sobre a ideia da complexidade como variável determinante da escolha das 
projetos. 


\section{CONCEITO DE PROJETO}

Uma definição frequentemente utilizada para o conceito de projeto - a definição convencional - é a que se encontra no Guia do PMBOK: um empreendimento temporário realizado para criar um produto, serviço ou resultado singular (PMI, 2008). A história dos projetos, na realidade, coloca em cheque a ideia de "temporário". A Catedral de Colônia foi construída do século XIII ao XVIII. Duração limitada, 600 anos? Diversos fatores podem ter contribuído para esse uso exagerado do tempo, mas a incompetência não é um deles. Os arquitetos sabiam fazer os desenhos, que seguiam modelos básicos; havia mão-de-obra especializada, que dominava as técnicas de construção; princípios e dispositivos de recrutamento, seleção, remuneração e organização de equipes eram usados regularmente; havia matéria-prima, ferramentas, meios de transporte... enfim, estavam disponíveis todos os recursos que já vinham sendo usados desde o nascimento da construção civil (DU COLOMBIER, 1973). Uma explicação plausível é a falta de dinheiro. Quando não há fundos, um projeto deve ser interrompido, mas a abundância de fundos não significa necessariamente sucesso na dimensão do uso do tempo. Projeto, portanto, às vezes, é um empreendimento com começo e fim predefinidos, dependendo da disponibilidade de recursos financeiros.

O compromisso básico dos projetos é a realização do resultado, que responde a uma necessidade ou problema. Controlar tempo, custos, riscos e qualidade, assim como outras variáveis, é condição para isso. Projetos sempre mudam a situação presente (ARCHIBALD, 2003), porque criam inovações destinadas ao mercado (novos produtos), ou processos de renovação interna (implantação de novos sistemas). Tudo isso conduz a novas situações, que geram novos projetos, num ciclo que se repete.

Além da questão do tempo, outra crítica à definição convencional é seu reducionismo: todos os projetos são empreendimentos bem definidos, manejáveis com o uso de fórmulas - são one-shot projects. Mesmo que essa definição se aplique a projetos de engenharia civil de grande porte, é, pelo menos, questionável, quando se trata do desenvolvimento de novos produtos ou de criação de tecnologias e soluções possíveis, que não existem no presente ou que ainda são rudimentares. O problema ou necessidade está bem definido, mas a solução é conhecida apenas no plano teórico, ou dela se tem apenas uma ideia. Esse tipo de projeto, exemplificado pelo desenvolvimento dos aviões militares a jato, exige elevado componente de experimentação, tornando a gestão do prazo consequência e não précondição (SCRANTON, 2008/8). Esse prazo é variável dependente da complexidade técnica 
do projeto. Muito diferente da construção das catedrais: a solução era conhecida desde o início; o prazo era variável dependente do custo. Em resumo, a natureza técnica dos projetos coloca em cheque a definição convencional.

Não apenas a natureza técnica dos projetos mas também a intangibilidade de determinados esforços planejados - por exemplo, a mudança da cultura de uma organização ou o desenvolvimento de produtos totalmente novos - tornam relevante a discussão sobre a complexidade e a incerteza no mundo dos projetos. Quanto maiores a complexidade e a incerteza, maior o risco. Presumivelmente, os projetos mais incertos, complexos e arriscados exigem processos gerenciais diferentes daqueles que estão na condição oposta. 


\section{COMPLEXIDADE E INCERTEZA NO MUNDO DOS PROJETOS}

Há diversas formas de definir a complexidade no mundo dos projetos. Para começar, há a complexidade relacional, que é função dos comportamentos sociais de equipes e de redes de pessoas dentro e fora de um projeto. Esse conceito aplica-se igualmente a pequenos empreendimentos e a programas de grande porte, pois complexidade não é sinônimo de complicação ou de tamanho, mas consequência direta do envolvimento de pessoas. Seja na execução de tarefas, seja pelo impacto que o trabalho de um grupo tem sobre outros stakeholders, o grau de complexidade de um projeto está intimamente ligado às relações humanas (WEAVER, 2007). A complexidade relacional também se mede pela quantidade de stakeholders aliados e adversários do projeto. Quanto mais numerosas as partes interessadas, quanto mais desfavorável a situação criada pelos adversários, mais complexo é o projeto do ponto de vista relacional (D'HERBEMONT; CESAR, 1998).

A complexidade técnica está relacionada com o produto e o conhecimento necessário para fazê-lo. É avaliada de diversas maneiras: o grau de dificuldade em construir o produto (IRELAND, 2007), o número de funções que o produto deve desempenhar, o número de componentes do produto, o grau de novidade e o número de tecnologias envolvidas e integração/interação entre elas (TATIKONDA; ROSENTHAL, 2000). Adotando como referência o trabalho de Baccarini (1996), Williams (1999) também considera o número de componentes (tarefas, especialistas, subsistemas, partes etc.), bem como a interdependência ou conectividade entre eles, como critérios para medir a complexidade - que ele chama de estrutural - de um projeto.

No que diz respeito ao grau de dificuldade para fazer o produto, a complexidade técnica é similar à incerteza. A incerteza em uma tarefa, segundo Galbraith (1977), citado por Tatikonda e Rosenthal (2000, p. 36), é "a diferença entre a quantidade de informação necessária para realizar a tarefa e a quantidade de informação da qual a organização dispõe" Para Turner e Cochrane (1993), a incerteza nos projetos tem duas dimensões: métodos e objetivos. A incerteza de métodos existe quando não se sabe como proceder para alcançar os objetivos. A incerteza de objetivos, por outro lado, aparece quando os requisitos do projeto estão mal definidos no início da fase de execução. Quanto maior o desconhecimento, seja sobre os objetivos, seja sobre a forma de realizá-los, maiores a incerteza e o risco. 
Certos projetos são feitos para lidar com a incerteza. São projetos que têm alguma forma de experimentação ou de pesquisa do desconhecido. É o caso do desenvolvimento de novos produtos, da pesquisa de novos medicamentos e da exploração do espaço. Um exemplo específico é o desenvolvimento dos jatos militares, logo após a Segunda Guerra (SCRANTON, 2008). De forma geral, é o caso de qualquer projeto que seja realizado para resolver um problema cuja solução é desconhecida no início. Esses projetos sabe-se onde começam, mas não onde terminam e sequer se sabe se algum tipo de resultado vai ser conseguido. Tão grande é a incerteza, que a incapacidade de alcançar um resultado prático não é considerada fracasso, nem justificativa para interromper a pesquisa, mas uma forma de aprendizagem. Além disso, eventos imprevistos (como acidentes e interferências do poder público) podem retardar a conclusão do projeto. Quando o grau de incerteza é muito alto, porque o produto é totalmente novo, nem o tempo nem o custo podem ser calculados com precisão, e o risco aumenta proporcionalmente.

Outro fator que contribui para a complexidade e a incerteza é o grau de familiaridade com os projetos na organização. Há também uma escala para medir isso (Graham, 1989): em um extremo, a organização não tem nenhuma familiaridade; no outro, a familiaridade com as ferramentas e os requisitos da administração de projetos é muito elevada. Dentro dessa escala, a dificuldade aumenta de forma inversamente proporcional ao grau de familiaridade ou maturidade. No extremo mais baixo da escala, a organização não sabe como lidar com projetos. Não há tradição com o conceito, não há estruturas nem procedimentos orientados para projetos, não há gerentes de projetos - em resumo, não há maturidade em gerenciamento de projetos. Os projetos e as pessoas responsáveis por sua condução estão em território desconhecido e possivelmente hostil. No outro ponto da escala, a situação é totalmente inversa e os projetos são conduzidos dentro de condições mais favoráveis.

Pollack (2007) oferece uma revisão da literatura sobre gerenciamento de projetos, explicando que o positivismo e o realismo serviram de base para o desenvolvimento desse tema. Significa dizer que o gerenciamento de projetos tradicional caracteriza-se por ser uma atividade marcada pela premeditação e funcionalismo com ênfase em técnicas reducionistas e de controle.

Segundo Weaver (2007), esse paradigma tradicional tem validade se os graus de conhecimento e entendimento do produto final são elevados, como nos projetos de engenharia e construção que evoluem de projetos de pesquisa. À medida que aumenta o domínio sobre o produto e diminui o grau de incerteza sobre como fazê-lo, as versões subsequentes tornam-se 
PUC Minas E\&G - REVISTA ECONOMIA E GESTÃO

projetos de desenvolvimento ou projetos de construção com pequenos aprimoramentos sucessivos. Podem-se, então, aplicar as técnicas de desenvolvimento rápido de projetos, que permitem criar um novo automóvel, por exemplo, em três anos ou menos. A definição consagrada pelo PMI passa a ter validade, nas condições em que o produto final é conhecido no início, e o prazo pode ser calculado com precisão, assim como o orçamento.

O paradigma tradicional torna-se questionável quando o produto final ainda deve ser descoberto, muda ao longo do tempo ou a natureza do trabalho se modifica. Nesses casos, a complexidade do empreendimento aumenta significativamente. Ireland (2007) apresenta estas variáveis que podem adicionar complexidade ao gerenciamento de projetos: arranjos financeiros que devem ser feitos com diferentes agentes para preencher necessidades momentâneas de capital sem o devido compromisso com prazos; estrutura gerencial formada por mais de uma empresa; ausência de profissionais com as habilidades necessárias; e mudanças de pessoas durante o projeto. 


\section{PROCESSO DE ADMINISTRAÇÃO DO PROJETO}

Não há uma teoria unificada e universalmente aceita sobre o que seja o processo da administração de projetos (SMYTH; MORRIS, 2007). Sendo inerentemente multidisciplinar, é um processo que se aplica desde a engenharia tradicional até a sistemas de informação, passando por áreas diversas como a sociologia e outras ciências comportamentais, sempre que uma abordagem sob encomenda é necessária. A gestão de projetos pode ser explorada de diversas formas, por diferentes clientes e stakeholders, públicos e privados, internos e externos, refletindo suas necessidades, sua cultura e seus valores, atingindo as mais diversas possibilidades de interesses, como problemas sociais e ambientais ou objetivos estratégicos. Essa amplitude exige o uso e justifica a existência das mais diversas ferramentas. (SMYTH, 2009).

Neste trabalho, a administração de um projeto é entendida como o processo de tomar decisões para realizar atividades temporárias, com o objetivo de fornecer um ou mais resultados. É "uma abordagem inovadora e específica que permite estruturar metódica e progressivamente uma realidade para a qual ainda não há equivalente exato (AFNOR, 1991)": um processo gradativo e determinado de construção de uma situação futura, com diferentes graus de certeza. Presumivelmente, os projetos de pesquisa e os de desenvolvimento de produtos com base em novas tecnologias têm grau mais elevado de incerteza, complexidade e risco que os de construção, por exemplo. (SCRANTON, 2008/8).

A tarefa básica da administração de projetos é assegurar a orientação do esforço para o resultado (TURNER, 1999). Controlar custos, prazos, riscos e outras variáveis é condição básica para a realização do resultado pretendido.

A aplicação dos princípios da administração de projetos depende tanto da natureza intrínseca da situação quanto de escolha consciente: projeto é uma atividade que alguém decide que é projeto. 


\section{CAIXA DE FERRAMENTAS}

As decisões que integram o processo de administrar definem a forma de conduzir o projeto: planejamento, organização, execução, controle e conclusão das atividades são as principais. As decisões aplicam-se a variáveis (ou áreas do conhecimento) que retratam os recursos e o contexto de qualquer projeto: escopo, prazo, custos, riscos, condições sociais e ambientais e assim por diante (PMI, 2008). A cada uma dessas áreas do conhecimento correspondem diferentes ferramentas ou técnicas. As ferramentas ou técnicas são procedimentos qualitativos e quantitativos que auxiliam o processo decisório de administrar o projeto. Ao tomar decisões sobre escopo, o gerente do projeto pode usar a WBS; ao tomar decisões sobre tempo, pode usar o gráfico de Gantt e assim por diante.

Essas técnicas e outras, estudadas no projeto de pesquisa relatado neste documento, estão relacionadas e referenciadas a seguir, associadas a cada uma das áreas do conhecimento: escopo, tempo, custos etc. Em sua maioria, são descritas na literatura. Pequeno número delas faz parte da experiência empírica e, dada sua simplicidade, dispensam referências.

\subsection{Escopo}

O conceito de escopo refere-se ao produto do projeto. O gerenciamento do escopo abrange os processos que realizam o trabalho necessário para completar o projeto com êxito: definição inicial dos objetivos, especificação das entregas etc.

a) Declaração do escopo (definição inicial dos objetivos do projeto) (PMI, 2008; HARVARD BUSINESS SCHOOL, 1997).

b) Dicionário da WBS (descrição detalhada dos níveis, deliverables e pacotes de trabalho da WBS) (PMBOK, 2008).

c) Estrutura analítica do projeto - WBS (PMI, 2008; HARVARD BUSINESS SCHOOL, 1997; IPMA, 2006; IEEE, 1998; CLELAND; IRELAND, 2000).

d) Diagrama (organograma) de tarefas (tipo WBS Pro) (CRITICAL TOOLS, 2009; PMI, 2008).

e) Gestão da configuração (definição e alterações do produto) (SAMARAS; CZERWINSKI, 1971; SHTUB; BARD; GLOBERSON, 1994). 
f) Project charter (termo de abertura, carta de missão do projeto) (PMI, 2008; IPMA, 2006).

\subsection{Prazos ou tempo}

O tempo e os prazos referem-se à duração do projeto e de suas fases. O gerenciamento do tempo compreende os processos necessários para realizar o projeto no prazo desejado - identificação, sequenciamento e estimativa de duração das atividades, bem como o controle de seu andamento.

a) Gráfico de Gantt (e variações) (HARVARD BUSINESS SCHOOL, 1997; BERGEN, 1986; CLELAND; KING, 1983; SHTUB; BARD; GLOBERSON, 1994).

b) Gráficos de rede (tipo PERT-CPM) (BERGEN, 1986; CLELAND; KING, 1983; SHTUB; BARD; GLOBERSON, 1994; PMI, 2008).

c) Métodos e análises da corrente crítica (PMI, 2008; GOLDRATT, 1997; LECHLER, RONEN; STOHR, 2005; GOLDRATT; COX, 1997; MYRTVEIT; STENSRUD, 1999).

\subsection{Custos}

Os custos referem-se aos recursos financeiros necessários para executar as atividades do projeto. O gerenciamento dos custos compreende os processos necessários para planejar e controlar o orçamento dos recursos do projeto.

a) Earned Value Management - EVM (curvas S e cálculo de variações de custo e prazos) (PMI, 2005; VARGAS, 2002; VARGAS, 2003; FLEMING; KOPPELMAN, 2005; GEROSA; CAPODIFERRO, 2009).

b) Estudo de viabilidade econômica (investimento e retorno) (BERGEN, 1986; COHEN; ZINBARG, 1967; SHTUB, BARD; GLOBERSON, 1994).

c) Nivelamento de recursos (histograma dos recursos ao longo do projeto) (PMI, 2008).

d) Projeto com objetivo de custo global (project life cycle costing) (ROUANET, 2000; WHITE; OSTWALD, 1976).

e) Técnicas de estimativa de custos (analógica, paramétrica, bottom up etc.) (PARK, 1999; PMI, 2008; CARVALHO; RABECHINI, 2009). 


\subsection{Qualidade}

O gerenciamento da qualidade compreende os processos necessários para garantir o atendimento das necessidades que deram origem ao projeto, abrangendo tanto o desempenho do produto quanto o do projeto.

a) Análise de valor (PARK, 1999).

b) Casa da qualidade (voz do consumidor transformada em especificações) (MCLAUGHLIN; STRATMAN, 1997; MEREDITH; SHAFER, 1999).

c) Diagrama de Ishikawa (análise de causa e efeito) (SCAVARDA et al., 2006).

d) Diagrama de Pareto (frequência de desvios e erros) (DESSLER, 2004, MEREDITH; SCOTT, 1999).

\subsection{Recursos humanos}

Os recursos humanos envolvem a gestão dos integrantes da equipe do projeto, sua designação para as diferentes tarefas, sua organização, avaliação de desempenho, estilos de liderança, de motivação etc.

a) Matriz linear de responsabilidades (organograma linear) (BACHY; HAMERI, 1997).

b) Organograma do projeto (gráfico da organização da equipe do projeto) (HYVÄRI, 2006; PMI, 2008).

c) Técnicas de gestão de conflitos (retirada, combate, delegação, compromisso, consenso etc.) (ZUTSHI; SOHAL, 2004).

d) Técnicas de team building (criatividade, condução de reuniões, comunicação, tomada de decisão, esclarecimento de papéis etc.) (LOO, 2003).

\subsection{Comunicações}

O gerenciamento das comunicações trata dos intercâmbios de informações necessárias para o gerenciamento do projeto e dos meios utilizados para registrá-las e transmiti-las. 
PUC Minas E\&G - REVISTA ECONOMIA E GESTÃO

a) Plano de comunicação (identificação dos stakeholders e das formas de documentação e transmissão da informação necessária e produzida pelo projeto) (CARVALHO; RABECHINI, 2009).

b) Prestação de contas (reporting) (relatórios e apresentações sobre o andamento e os resultados) (PMI, 2008).

c) Reuniões de acompanhamento (encontros periódicos da equipe do projeto e de outras partes interessadas)

\subsection{Riscos}

O gerenciamento dos riscos compreende os processos necessários para lidar com as ameaças e os eventos desfavoráveis ao projeto - identificação, análise e plano de ações para enfrentar os riscos.

a) Planejamento das respostas aos riscos (evitar, mitigar, transferir, aceitar) (PMI, 2008; SHTUB; BARD; GLOBERSON, 1994).

b) Planos de contingência (para o caso de algum risco acontecer) (CLELAND; IRELAND, 2000).

c) Matriz de análise dos riscos (avaliação do impacto e probabilidade dos riscos) (COX, 2008; BERGEN, 1986; CLELAND; IRELAND, 2000; CLELAND; KING, 1983).

d) FMEA-FMECA (avaliação das falhas potenciais e riscos do produto) (SEMATECH, 1992).

\subsection{Suprimentos ou fornecimentos}

Os suprimentos e fornecimentos constituem processos necessários para gerenciar os bens e serviços fornecidos por outras organizações.

a) Chamada de propostas, licitação (participação em concorrências) (PMI, 2008).

b) Técnicas de negociação (CLELAND; IRELAND, 2000).

c) Apoio logístico integrado (o apoio necessário nas fases de desenvolvimento e operação ao longo do ciclo de vida de um produto) (KUMAR; CROCKER; KNEZEVIC; ELHARAM, 2000).

\subsection{Responsabilidade social}


A responsabilidade social trata da análise, do planejamento e da execução de atividades de proteção do ambiente, da sociedade e da integridade das pessoas.

a) Análise de viabilidade ambiental e social (estudo prévio dos impactos causados pelo projeto sobre o ambiente e a sociedade) (O'SHAUGNESSY, 1992).

b) Análise do ciclo de vida (estudo dos impactos ambientais e sociais do projeto ao longo de seu ciclo de vida) (CLELAND; IRELAND, 2000; REBITZER; BUXMANN, 2005; SCHALTEGGER; STURM，1992; SCHALTEGGER; STURM，1998; ZUTSHI; SOHAL, 2004).

c) Estudos de impacto ambiental e social (estudo detalhado dos impactos ambientais e sociais do projeto) (BROCHE; CAPRON; QUAIREL-LANOIZELLE, 2005; LAWRENCE et al., 2002; ZUTSHI; SOHAL, 2004).

d) Saúde, meio ambiente e segurança (SMS) ou Environment, Health and Safety (EHS) (estudo integrado dos impactos do projeto sobre o ambiente e a integridade físicas das pessoas) (IPMA, 2006; SHEN; WALKER, 2001; WILKINSON; DALE, 1999).

\subsection{Conhecimento}

O planejamento e a implementação de atividades visam identificar, compilar, organizar e guardar o conhecimento gerado pelos projetos, bem como proteger o sigilo e a propriedade intelectual contida nesses conhecimentos.

a) Análise e registro de lições aprendidas (durante e ao final do projeto) (CCTA, 1999; IPMA, 2006; PMI, 2008)

b) Mapeamento de competências (das pessoas, para alocação aos projetos) (CCTA, 1999; MARBOT, 2007).

c) Gestão da propriedade intelectual (DAVENPORT; DE LONG; BEERS, 1998).

\subsection{Integração}

São necessários processos que assegurem a integração de todos os elementos do projeto, por meio da elaboração e execução de um plano de projeto.

a) Avaliação do contexto do projeto (condições organizacionais, culturais, geográficas etc. específicas da realização do projeto) (PMI, 2008; IPMA, 2006; IEEE, 1998).

b) Carta de aceitação pelo cliente (aprovação parcial ou final do projeto / produto) (PMI, 2008). 
PUC Minas E\&G - REVISTA ECONOMIA E GESTÃO

c) Controle da execução (acompanhamento da execução do plano do projeto) (PMI, 2008)

d) Kick-off meeting (reunião de lançamento do projeto) (BIEDERMANN; SEIDEL, 2007; HAMBURGER, 1992).

e) Plano mestre do projeto (PMI, 2008).

f) Solicitação formal de mudanças (na configuração do produto, do escopo, do prazo, orçamento do projeto etc.) (PMI, 2008; KHAN, 2006).

g) Sponsor (indicação de um patrono ou apoiador do projeto na administração superior) (IPMA, 2006; CLELAND; IRELAND, 2000). 


\section{PROCEDIMENTOS}

Este estudo consistiu de um levantamento conduzido por meio de questionário com o formato apresentado na Figura 1.

Figura 1 - Formato do questionário

\begin{tabular}{|l|l|l|l|l|l|l|l|l|l|l|l|l|l|l|l|l|l|}
\hline FERRAMENTAS & \multicolumn{1}{|l|}{ CONCEP } \\
PLANEJ & \multicolumn{7}{|l|}{ EXECUÇÃo } \\
\hline n. Ferramenta n (descrição) & N & R & O & F & S & N & R & O & F & S & N & R & O & F & S \\
\hline
\end{tabular}

Fonte: Elaborado pelos autores.

Esse formato retrata a premissa de um ciclo de vida com três fases para qualquer projeto. Aos respondentes se solicitou que indicassem a frequência de uso das 47 ferramentas propostas, em cada uma das três fases, assinalando suas respostas na escala do tipo Likert: nunca, raramente etc.

A amostra foi composta de forma intencional, e o levantamento produziu um total de 168 respostas, das quais 64 fornecidas por profissionais da construção civil e 104 por profissionais de outras áreas. Foram convidados a preencher o questionário, de forma exclusivamente presencial, estudantes de cursos de extensão universitária de gerenciamento de projetos. Os integrantes da amostra deveriam responder com base em sua experiência pessoal, sem se preocupar em retratar as práticas de sua organização. 


\section{DADOS}

Os dados obtidos no levantamento estão retratados nas tabelas e nos gráficos apresentados a seguir, que são autoexplicativos. Neste trabalho, serão apresentados os dados relativos a apenas cinco das 11 áreas do conhecimento pesquisadas, porque são as que aparecem na Tabela 1. Essa tabela, abaixo, apresenta a frequência média das respostas SEMPRE e FREQUENTEMENTE nas três fases, comparando o setor de construção civil com os demais. As frequências de uso das ferramentas são colocadas em ordem decrescente, até a posição 10.

Tabela 1 - Comparação entre as frequências de construção civil e outros

\begin{tabular}{|c|c|c|c|c|c|}
\hline \multicolumn{3}{|c|}{ CONSTRUÇÃO CIVIL } & \multicolumn{3}{|c|}{ OUTROS } \\
\hline \# & $\begin{array}{l}\text { FERRAMENTAS DE } \\
\text { GERENCIAMENTO DE } \\
\text { PROJETOS }\end{array}$ & $\begin{array}{l}\text { Frequência } \\
\text { média }(\%)\end{array}$ & \# & $\begin{array}{l}\text { FERRAMENTAS DE } \\
\text { GERENCIAMENTO DE } \\
\text { PROJETOS }\end{array}$ & $\begin{array}{l}\text { Frequência } \\
\text { média }(\%)\end{array}$ \\
\hline 1 & Reuniões de acompanhamento & 68,8 & 1 & Prestação de contas (reporting) & 59,6 \\
\hline 2 & Prestação de contas (reporting) & 62,5 & 2 & Reuniões de acompanhamento & 59,0 \\
\hline 3 & Gestão de contratos & 53,6 & 3 & Controle da execução & 51,3 \\
\hline 4 & Declaração do escopo & 51,6 & 4 & Organograma do projeto & 42,3 \\
\hline 5 & Controle da execução & 50,5 & 5 & Declaração do escopo & 41,3 \\
\hline 6 & Organograma do projeto & 49,0 & 6 & Carta de aceitação pelo cliente & 40,7 \\
\hline 7 & Carta de aceitação pelo cliente & 47,4 & 7 & Gestão de contratos & 38,5 \\
\hline 8 & $\begin{array}{l}\text { Estrutura analítica do projeto - } \\
\text { EAP ou WBS }\end{array}$ & 45,8 & 8 & $\begin{array}{l}\text { Matriz linear de } \\
\text { responsabilidades }\end{array}$ & 36,9 \\
\hline 9 & Solicitação formal de mudanças & 44,8 & 9 & Sponsor & 36,5 \\
\hline 10 & $\begin{array}{l}\text { Técnicas de estimativa de } \\
\text { custos }\end{array}$ & 43,2 & 10 & Kick-off meeting & 34,1 \\
\hline 11 & $\begin{array}{l}\text { Avaliação do contexto do } \\
\text { projeto }\end{array}$ & 40,6 & 11 & Gráfico de Gantt & 33,7 \\
\hline 12 & Diagrama de tarefas & 39,6 & 12 & $\begin{array}{l}\text { Análise e registro de lições } \\
\text { aprendidas }\end{array}$ & 33,7 \\
\hline 13 & Gráfico de Gantt & 39,6 & 13 & Planos de contingência & 33,0 \\
\hline 14 & Técnicas de team building & 38,0 & 14 & Técnicas de negociação & 33,0 \\
\hline 15 & Kick-off meeting & 34,9 & 15 & $\begin{array}{l}\text { Avaliação do contexto do } \\
\text { projeto }\end{array}$ & 32,5 \\
\hline
\end{tabular}




\subsection{Escopo}

Nesta categorização, representada na Tabela 2, a seguir, considerou-se o somatório das respostas SEMPRE e FREQUENTEMENTE para a análise do emprego das ferramentas aplicadas à gestão do ESCOPO dos projetos, nas três fases, nos dois grupos escolhidos.

Tabela 2 - Uso das ferramentas de escopo nos dois grupos

\begin{tabular}{|c|c|c|c|c|c|c|}
\hline \multirow{2}{*}{ - } & \multicolumn{2}{|c|}{$\begin{array}{l}\text { CONCEPÇÃO/ } \\
\text { PLANEJAM. }\end{array}$} & \multicolumn{2}{|c|}{ EXECUÇÃO } & \multicolumn{2}{|c|}{$\begin{array}{l}\text { ENCERRA- } \\
\text { MENTO }\end{array}$} \\
\hline & Civil & Outros & Civil & Outros & Civil & Outros \\
\hline Avaliação do contexto do projeto & 46,9 & 36,5 & 51,6 & 37,9 & 23,4 & 23,1 \\
\hline Project charter & 29,7 & 25,0 & 25,0 & 20,2 & 20,3 & 17,3 \\
\hline Declaração do escopo & 60,9 & 41,3 & 51,6 & 46,2 & 42,2 & 36,5 \\
\hline Estrutura analítica do projeto - EAP (WBS) & 57,8 & 30,8 & 50,0 & 37,5 & 29,7 & 20,2 \\
\hline Dicionário da WBS & 28,1 & 25,0 & 29,7 & 15,4 & 18,8 & 9,6 \\
\hline Análise do ciclo de vida & 9,4 & 13,5 & 12,5 & 14,4 & 9,4 & 10,6 \\
\hline
\end{tabular}

\subsection{Tempo}

Nesta categorização, representada na Tabela 3, considerou-se o somatório das respostas SEMPRE e FREQUENTEMENTE para a análise do emprego das ferramentas aplicadas à gestão do TEMPO ou PRAZOS dos projetos, nas três fases, nos dois grupos escolhidos.

Tabela 3 - Uso das ferramentas de tempo nos dois grupos

\begin{tabular}{|c|c|c|c|c|c|c|}
\hline \multirow{2}{*}{ - } & \multicolumn{2}{|c|}{$\begin{array}{l}\text { CONCEPÇÃO/ } \\
\text { PLANEJAM. }\end{array}$} & \multicolumn{2}{|c|}{ EXECUÇÃO } & \multicolumn{2}{|c|}{$\begin{array}{l}\text { ENCERRA- } \\
\text { MENTO }\end{array}$} \\
\hline & CIVIL & Outros & CIVIL & Outros & CIVIL & Outros \\
\hline Diagrama de tarefas & 46,9 & 30,8 & 43,8 & 39,4 & 28,1 & 20,2 \\
\hline Gráfico de Gantt & 40,6 & 26,0 & 46,9 & 46,2 & 31,3 & 28,8 \\
\hline Gráficos de rede & 14,1 & 19,2 & 21,9 & 16,3 & 10,9 & 11,5 \\
\hline Métodos e análises da corrente crítica & 20,3 & 22,1 & 23,4 & 11,5 & 12,5 & 5,8 \\
\hline Nivelamento de recursos & 35,9 & 23,1 & 29,7 & 23,3 & 25,0 & 10,7 \\
\hline
\end{tabular}




\subsection{Custos}

Nesta categorização, representada na Tabela 4, considerou-se o somatório das respostas SEMPRE e FREQUENTEMENTE para a análise do emprego das ferramentas aplicadas à gestão dos CUSTOS dos projetos, nas três fases, nos dois grupos escolhidos.

Tabela 4 - Uso das ferramentas de custos nos dois grupos

\begin{tabular}{|c|c|c|c|c|c|c|}
\hline- & \multicolumn{2}{|c|}{$\begin{array}{l}\text { CONCEPÇÃO/ } \\
\text { PLANEJAM. }\end{array}$} & \multicolumn{2}{|c|}{ EXECUÇÃ̃ } & \multicolumn{2}{|c|}{$\begin{array}{l}\text { ENCERRA- } \\
\text { MENTO }\end{array}$} \\
\hline - & CIVIL & Outros & CIVIL & Outros & CIVIL & Outros \\
\hline Técnicas de estimativa de custos & 53,1 & 27,9 & 42,2 & 31,7 & 34,4 & 20,4 \\
\hline Estudo de viabilidade econômica & 34,4 & 30,1 & 29,7 & 22,1 & 21,9 & 22,1 \\
\hline Projeto (design) para objetivo de custo global & 25,0 & 18,3 & 25,0 & 16,3 & 20,3 & 12,5 \\
\hline EVM = Earned Value Management & 26,6 & 15,4 & 31,3 & 13,5 & 18,8 & 10,6 \\
\hline Análise de valor & 29,7 & 17,3 & 29,7 & 12,5 & 21,9 & 10,6 \\
\hline
\end{tabular}

Fonte: Elaborado pelos autores.

\subsection{Riscos}

Nesta categorização, exposta na Tabela 5, considerou-se o somatório das respostas SEMPRE e FREQUENTEMENTE para a análise do emprego das ferramentas aplicadas à gestão dos RISCOS dos projetos, nas três fases, nos dois grupos escolhidos.

Tabela 5 - Uso das ferramentas de riscos nos dois grupos

\begin{tabular}{|c|c|c|c|c|c|c|}
\hline \multirow{2}{*}{$\begin{array}{l}- \\
- \\
-\end{array}$} & \multicolumn{2}{|c|}{$\begin{array}{l}\text { CONCEPÇÃO/ } \\
\text { PLANEJAM. }\end{array}$} & \multicolumn{2}{|c|}{ EXECUÇÃO } & \multicolumn{2}{|c|}{$\begin{array}{l}\text { ENCERRA- } \\
\text { MENTO }\end{array}$} \\
\hline & CIVIL & Outros & CIVIL & Outros & CIVIL & Outros \\
\hline Matriz de análise dos riscos & 21,9 & 28,8 & 26,6 & 35,6 & 17,2 & 28,8 \\
\hline FMEA-FMECA & 7,8 & 16,3 & 14,1 & 16,3 & 10,9 & 12,5 \\
\hline Planos de contingência & 23,4 & 31,7 & 28,1 & 38,5 & 23,4 & 28,8 \\
\hline Planejamento das respostas aos riscos & 21,9 & 22,1 & 21,9 & 35,6 & 15,6 & 26,9 \\
\hline
\end{tabular}


Fonte: Elaborado pelos autores.

\subsection{Comunicações}

Nesta categorização, exposta na Tabela 6, considerou-se o somatório das respostas SEMPRE e FREQUENTEMENTE para a análise do emprego das ferramentas aplicadas à gestão das Comunicações dos projetos, nas três fases, nos dois grupos escolhidos.

Tabela 6 - Uso das ferramentas de comunicações nos dois grupos

\begin{tabular}{|c|c|c|c|c|c|c|}
\hline \multirow{2}{*}{$\begin{array}{l}- \\
- \\
-\end{array}$} & \multicolumn{2}{|c|}{$\begin{array}{l}\text { CONCEPÇÃO/ } \\
\text { PLANEJAM. }\end{array}$} & \multicolumn{2}{|c|}{ EXECUÇÃO } & \multicolumn{2}{|c|}{$\begin{array}{l}\text { ENCERRA- } \\
\text { MENTO }\end{array}$} \\
\hline & CIVIL & Outros & CIVIL & Outros & CIVIL & Outros \\
\hline Kick-off meeting & 42,2 & 47,1 & 35,9 & 28,8 & 26,6 & 26,2 \\
\hline Sponsor & 25,0 & 33,7 & 23,4 & 39,4 & 20,3 & 36,5 \\
\hline Plano de comunicação & 31,3 & 26,9 & 37,5 & 39,4 & 26,6 & 29,8 \\
\hline Prestação de contas (reporting) & 51,6 & 42,3 & 75,0 & 73,1 & 60,9 & 63,5 \\
\hline Reuniões de acompanhamento & 67,2 & 45,2 & 76,6 & 75,0 & 62,5 & 56,7 \\
\hline
\end{tabular}

Fonte: Elaborado pelos autores. 


\section{ANÁLISE E REFLEXÕES}

Apesar da pequena quantidade de dados, há evidências sugestivas nessas tabelas.

Fazendo-se referência à Tabela 1, as ferramentas são mais usadas na construção civil do que nas outras áreas - tecnologia da informação, consultoria, desenvolvimento de produtos etc. As ferramentas de natureza mais técnica - WBS, gráfico de Gantt, orçamento - são menos usadas que outras práticas de natureza relacional - reuniões, acompanhamento de contratos e prestação de contas. Há substancial similaridade entre os dois grupos, assim como sutis diferenças na ordenação das ferramentas.

As tabelas específicas oferecem maior clareza em cada um dos dois casos, bem como mostram as diferenças entre eles.

De acordo com a Tabela 2, nos dois grupos, a declaração de escopo e a WBS são as ferramentas de escopo mais usadas. Também se observa que o uso dessas ferramentas decresce à medida que o projeto avança.

Na Tabela 3, revela-se que o gráfico de Gantt é a ferramenta de planejamento mais usada, enquanto os métodos de redes são pouco usados. Os dados, aqui, sustentam a ideia de que o cronograma clássico é muito mais usado do que as redes. Apesar de todo o tempo dedicado às redes nos programas de treinamento em gerenciamento de projetos, os praticantes ainda preferem uma ferramenta mais antiga. Presumivelmente, não se trata de deficiência das práticas mas de facilidade de uso da ferramenta de Gantt.

NaTabela 4, percebe-se que a construção civil usa ferramentas de custos muito mais frequentemente do que os integrantes do outro grupo. Superficialmente, as ferramentas de gestão dos riscos são pouco usadas nos dois grupos, pelos dados da Tabela 5. Nota-se, no entanto, que as ferramentas dessa área do conhecimento são mais usadas em P\&D, TI e outras áreas de aplicação do que na construção civil. Presumivelmente, isso indica que, sendo a construção civil uma área de aplicação muito mais antiga e consolidada que as demais, esta tem projetos com menor incerteza, enquanto nas outras se justifica maior preocupação com a estimativa dos riscos. Ainda que com base em reduzido volume de dados, há indícios de diferenças entre os dois grupos. As técnicas mais orientadas para o tratamento da incerteza e da complexidade são usadas com maior frequência no grupo das áreas de tecnologias mais recentes. O reforço dessa conclusão depende de maior quantidade de dados, cuja obtenção faz parte de um novo projeto de investigação. 
Finalmente, é evidente, pela Tabela 6 , que as ferramentas (e o processo) da comunicação apresentam os números mais altos de utilização.

É arriscado, porém lícito, supor que a complexidade e a incerteza dos projetos explicam os dados sobre a comunicação, porém de maneira diferente em cada um dos dois casos. Na área de construção, provavelmente, é mais importante a comunicação do que as demais ferramentas porque a experiência com projetos, por parte dos especialistas, assim como a familiaridade, por parte dos usuários, não exigem muito mais do que a planta, o orçamento e os estudos de viabilidade econômica. Assim, a comunicação torna-se o processo básico não apenas na fase de planejamento mas também ao longo de todo o projeto. No caso dos projetos de desenvolvimento de produtos e tecnologia da informação, a incerteza e, portanto, a complexidade, são mais elevadas. Na construção civil, o projeto começa com um produto totalmente desenhado; nas demais áreas, os projetos começam com problemas e o produto deve ser desenhado e construído passo a passo. Projetos de consultoria, de desenvolvimento de sistemas, e mesmo os mais sofisticados de construção civil, como os trens de alta velocidade, requerem um pré-projeto de diagnóstico e estudo de viabilidade. Somente depois desse pré-projeto é possível preparar um plano com escopo, tempo e custos previstos. O escopo, no entanto, muda ao longo dos projetos com algum grau de experimentação, como é claramente o caso da pesquisa e do desenvolvimento de produtos. Esses problemas são resolvidos com processos de comunicação, não com processos administrativos e técnicas formais. Em resumo, a complexidade e a incerteza são dimensões importantes para explicar a forma como são administrados os projetos.

Os conceitos e as técnicas da administração de projetos constituem uma tecnologia que permite às pessoas resolver problemas de tempo, trabalho e uso de recursos, em condições de complexidade e incerteza que variam de um caso para outro. Sua utilização em situações práticas é calibrada não apenas pelo grau de complexidade e incerteza mas também pela experiência e domínio dessa tecnologia pelo gerente de projetos e por outros stakeholders. A análise da relação entre experiência, tipo de projeto e uso de ferramentas está além do escopo deste trabalho, mas permanece com desafio para outros estudos. 


\section{REFERÊNCIAS}

AFNOR Norme X50-105 Août, 1991. Disponível em: 〈http://www.afnor.org>. Acesso em: nov. 2009.

ARCHIBALD, R. D. Managing High-Tecnology Programs and Projects. 3. ed. New York: John Wily \& Sons Inc., 2003.

BACCARINI, D. The concept of project complexity-a review. International Journal of Project Management, v. 14, n. 4, p. 201-204, 1996.

BACHY, G.; HAMERI, A. P. What to be implemented at the early stage of a large-scale project. International Journal of Project Management, v. 15, n. 4, p. 211-2181997.

BESNER, C.; HOBBS, B. A Contextual Assessment of Project Management Practice: Variation by knowledge area, project type and phase. Project Perspectives, v. 29, p. 10-15, 2008 .

BIEDERMANN, M.; SEIDEL, A. Aller Anfang ist schwer - Problem - und zieldefinition in der organisationsberatung. Gruppendynamik und Organisationsberatung, v. 38, n. 2007.

BROCHE, K.; CAPRON, M.; QUAIREL-LANOIZELLE, F. Grands projets et exercice de la responsabilité globale: les études d'impact social. Management Prospective Editions, Revue management et avenir, n. 3, p. 121-151, 2005/1.

CARVAlHO, M. M.; RABECHINI, R. Jr. Construindo Competências para Gerenciar Projetos: teoria e casos. 2. ed. São Paulo: Editora Atlas, 2009.

CENTRAL COMPUTERS \& TELECOMMUNICATIONS AGENCY - CCTA. Managing Successful Projects with PRINCE 2 - Electronic Manual. Key Skills, 1999.

CLELAND, D. I.; IRELAND, L. R. Project manager's portable handbook. New York: McGraw Hill, 2000.

CLELAND, D. I.; KING, W. Industrial project management: addresses, essays, lectures. New York: Van Nostrand Reinhold Co., 1983.

COHEN, J. B.; ZINBARG, E. D. Investment analysis and portfolio management. Homewood, Ill.: R.D. Irwin, 1967.

COX Jr., L. A.. What's wrong with risk matrices. Risk Analysis, v. 28, n. 2, p. 497-512, 2008.

CRITICAL TOOLS. Disponível em<http://www.criticaltools.com/wbschartprosoftware.htm> Acesso em: nov. 2009.

DAVENPORT, T. H.; DE LONG, D. W.; BEERS, M. C. Successful Knowledge Management Projects. Sloan Management Review, v. 39, p. 43-57, 1998.

DESSLER, G. Management. $3^{\text {rd }}$ edition. Upper Saddle River, New Jersey: Pearson Prentice Hall, 2004.

D'HERBEMONT, O.; CESAR, B. Managing Sensitive Projects. London: Macmillan Press, 1998.

DU COLOMBIER, P. Les chantiers des cathedrals. Paris: Picard, 1973. 
FLEMING, Q. W.; KOPPELMAN, J. M. Earned Value Project Management, $3{ }^{\text {rd }}$ edition. Newton Square, PA: Project Management Institute, 2005.

GEROSA, S.; CAPODIFERRO, C. Earned Value Management (EVM) Techniques for Engineering and Prototype Production Activities. PM WORLD TODAY - Second Edition - July, 2009.

GOLDRATT, E. M. Critical Chain. Great Barrington, MA: The Northriver Press Publishing Corporation, 1997.

GOLDRATT, E. M.; COX, J. The Goal: a process of on going improvement. Great Barrington, MA: The Northriver Press Publishing Corporation, 1997.

GRAHAM, R. J. Project Management as if People Mattered. Primavera Press, 1989.

HAMBURGER, D. Project kick-off: getting the project off on the right foot. International Journal of Project Management, v. 10, n. 2, p. 115-122, 1992.

HARVARD BUSINESS SCHOOL. Project Management Manual. Boston: Harvard Business School, 1997.

HYVÄRI, I. Project management effectiveness in project-oriented business organizations. International Journal of Project Management, v. 24, n. 3, p. 216-225, 2006.

INSTITUTE OF ELECTRICAL AND ELECTRONICS ENGINEERS, THE - IEEE. IEEE Standard for Software Project Management Plans. New York: IEEE, 1998.

INTERNATIONAL PROJECT MANAGEMENT ASSOCIATION - IPMA. ICB - IPMA Competence Baseline, 2006. Disponível em:

<http://www.ipma.ch/certification/standards/Pages/ICBV3.aspx > Acesso em: nov. 2009.

IRELAND, L. Project Complexity: A Brief Exposure to Difficult Situations. PrezSez 10, 2007. Disponível em: 〈http://www.asapm.org/asapmag/articles/PrezSez10-07.pdf〉. Acesso em: nov. 2009.

KHAN, A. Project scope management. Cost Engineering, Morgantown, West Virginia, v. 48, n. 6, p. 12-16, 2006.

KUMAR, D.; CROCKER, J.; KNEZEVIC, J.; EL-HARAM, M. Reliability, maintenance and logistic support - a lifecycle approach. Norwell: Kluwer Academic Publishers, 2000.

LAWRENCE, L.; ANDREWS, D.; RALPH, B.; FRANCE, C. Identifying and assessing environmental impacts: investigating ISO 14001 approaches. TQM Magazine, v. 14, n. 1, p. 43-50, 2002.

LECHLER, T. G.; RONEN, B.; STOHR, E. A. Critical Chain: A New Project Management Paradigm or Old Wine in New Bottles? Engineering Management Journal, n. 17, Dec. 2005.

LOO, R. Assessing "team climate" in project teams. International Journal of Project Management, v. 21, n. 7, p. 511-517, 2003.

MARBOT, É. Compétences: la reference le la gestion des employs. THÉVENET, M.; DEJOUX, C.; MARBOT, É.; BENDER, A.-F. Fonctions RH - Politiques, metiers et outils des ressources humaines. Paris: Pearson Education France, 2007.

MCLAUGHLIN, C. P.; STRATMAN, J. K. Improving the quality of corporate technical planning: dynamic analogues of QFD. R\&D Management, v.27, n. 3, p. 269-279 1997. 
MEREDITH, J. R.; SHAFER, S. M. Operations management for MBAs. New York: John Wiley \& Sons, 1999.

MYRTVEIT, I.; STENSRUD, E. Controlled Experiment to Assess the Benefits of Estimating with Analogy and Regression Models. IEEE transactions on software engineering, v. 25, n. 4, jul./ago, 1999.

O'SHAUGNESSY, W. La faisabilité de projet. Trois-Rivières, Québec: Les Éditions SMG, 1992.

PARK, R. Value Engineering: a plan for invention, 1999. Disponível em: <http://www.value-eng.org/>. Acesso em: nov. 2009.

POLLAK, J. The changing paradigms of project management. International Journal of Project Management, n. 25, p. 266-274, 2007.

PROJECT MANAGEMENT INSTITUTE. A Guide to the Project Management Body of Knowledge (PMBOK Guide), $4^{\mathrm{TH}}$ edition. Newton Square, PA: PMI, 2008.

PROJECT MANAGEMENT INSTITUTE. Practice Standard for Earned Value Management. Newton Square, PA: PMI, 2005.

PROJECT MANAGEMENT INSTITUTE. Practice Standard for Work Breakdown Structures, 2nd edition. Newton Square, PA: PMI, 2006.

REBITZER, G.; BUXMANN, K. The role and implementation of LCA within life cycle management at Alcan. Journal of Cleaner Production, n. 13, p. 1327-1335, 2005.

ROUANET, D.2000.Disponível em: <http://www.afc-

cca.com/archives/docs_congres/congres2000/Angers/Fichiers/ROUANET.pdf $>$. Acesso em: nov. 2009.

SAMARAS, T. T.; CZERWINSKI, F. L. Fundamentals of configuration management. New York: Wiley-Interscience, 1971.

SCAVARDA, A. J.; BOUZDINE-CHAMEEVA, T.; GOLDSTEIN, S.M.; HAYS, J. M. HILL, A. V. A methodology for constructing collective casual maps. Decision Sciences, v. 37 n. 2, p. 263-283, 2006.

SCHALTEGGER, S.; STURM, A. Erfolgskriterien ökologieorientierten Managements. Zfu v. 15, n. 2, p. 131-154, 1992.

SCHALTEGGER, S.; STURM, A. Eco-Efficient by Eco-Controlling. On the implementation of EMAS and ISO 14001. Hochschulverlag, Zurich, 1998.

SCRANTON, P.. Le management de projet. Nouvel objet de l'histoire d'entreprise. Revue française de gestion, n. 188-189, p. 161-173, 2008/8.

SEMATECH. 1992. Disponível em:

<http://ismi.sematech.org/docubase/document/0963beng.pdf >. Acesso em: nov. 2009.

SHEN, Y. J.; WALKER, D. H. T. Integrating OHS, EMS and QM with constructability principles when construction planning - A design and construct project case study. TQM Magazine, v. 13, n. 4, p.247-259, 2001.

SMYTH, H. J. Projects and Programs: Diversity of management, diversity of aims and interests. International Journal of Project Management, v. 27, n. 2, 2009. 
SMYTH, H. J.; MORRIS, P. W. G. An epistemological evaluation of research into projects and their management: methodological issues. International Journal of Project Management, v. 25, n. 4, p.423-436, 2007.

SHTUB, A.; BARD, J. F.; GLOBERSON, S. Project management: engineering, technology, and implementation. Englewood Cliffs, NJ: Prentice Hall, 1994.

TATIKONDA, M. V.; ROSENTHAL, S. R. Technology Novelty, Project Complexity, and Product Development Project Execution Success: A Deeper Look at Task Uncertainty in Product Innovation. IEEE Transactions on Engineering Management, v. 47, n. 1, fev. 2000 .

TURNER, J. R. The Handbook of Project Based Management. London: McGraw-Hill Publishing Company, 1999.

TURNER, J. R.; COCHCRANE, R. A. Goals and methods matrix: coping with projects with ill-defined goals and/or methods for achieving them. International Journal of Project Management, v.11, n. 2, p. 93-102, 1993.

VARGAS, R. V. Análise de valor agregado em projetos: revolucionando o gerenciamento de custos e prazos. Rio de Janeiro: Brasport Livros e Multimídia Ltda, 2002.

VARGAS, R. V. Manual prático do plano de projeto utilizando o PMBK 2000. Rio de Janeiro: Brasport Livros e Multimídia Ltda, 2003.

WEAVER, P. A simple view of 'complexity' in project management. WPM Week. Singapore, p. 14-16, nov. 2007.

WHITE, G. E., OSTWALD, P. F. Life cycle costing. Management Accounting January, n. 39, 40, 42, 1976.

WILLIAMS, T. M. The need for new paradigms for complex projects. International Journal of Project Management, v. 17, n. 5, p. 269-273, 1999.

WILKINSON, G.; DALE, B. G. Integrated management systems: an examination of the concept and theory. TQM Magazine, v. 11, n. 2, p. 95-104, 1999.

ZUTSHI, A.; SOHAL, A. S. Adoption and maintenance of environmental management systems: Critical success factors. Management of Environmental Quality, v. 15, n. 4, p. 399-419, 2004. 